
$\$$ Research Square
Preprints are preliminary reports that have not undergone peer review.
They should not be considered conclusive, used to inform clinical practice,
or referenced by the media as validated information.

\title{
Type I and li Pulmonary Atresia With Intact Ventricular Septum in Infants-10-year Experience in Initial Surgery at One Center
}

\section{Hailong Song}

Second Hospital of Hebei Medical University

\section{Ziying Chen ( $\sim 2683292687 @ q q . c o m$ )}

Second Hospital of Hebei Medical University

\section{Research Article}

Keywords: mechanical ventilation time, tricuspid valve $Z$ value, degree of tricuspid regurgitation, oxygen saturation

Posted Date: April 13th, 2021

DOI: https://doi.org/10.21203/rs.3.rs-403631/v1

License: (c) (i) This work is licensed under a Creative Commons Attribution 4.0 International License.

Read Full License

Version of Record: A version of this preprint was published at BMC Cardiovascular Disorders on March 17th, 2022. See the published version at https://doi.org/10.1186/s12872-022-02549-1. 


\section{Abstract}

Background: To explore the effect of initial surgery for type I and II pulmonary atresia with intact ventricular septum (PA/IVS).

Methods: Patients with type I and II PA/IVS undergoing initial surgery were enrolled, including type I with systemic to pulmonary (BT) shunt+patent ductus arteriosus (PDA) ligation+mosaic surgery (surgical A), BT shunt+PDA ligation+pulmonary valve incision without extracorporeal circulation (surgical B), type II with BT shunt+PDA ligation+right ventricular outflow tract (RVOT) incision+transpulmonary annulus patch (TP) (surgical C), and BT shunt+PDA ligation+RVOT incision+TP+artificial pulmonary valve (surgical D). Mechanical ventilation time (MVT), length of ICU stay, mortality rate, tricuspid Z value (TZ), tricuspid regurgitation (TR), McGoon ratio, oxygen saturation (SpO2), pulmonary transvalvular pressure (PTP), pulmonary regurgitation (PR), survival rate and re-operation rate were compared between surgical $A$ and $B$, and between surgical $C$ and $D$.

Results: After surgery, PR was greater by surgical $A$ than by surgical $B$ at 1 month $(P<0.05)$; lower TZ and McGoon ratio and greater PR, PTP and TR at 3 months $(P<0.05)$; lower SpO2 $(P<0.05)$, greater PTP at 6 months $(P<0.01)$; greater TR and PTP at 1 year $(P<0.05)$. MVT and length of ICU stay were longer by surgical $C$ than by surgical $D(P<0.05)$. There was greater $P R$ at discharge and 1 month $(P<0.01)$; greater TR and PR, lower McGoon ratio and SpO2 at 3 months $(P<0.05)$; lower TZ and PTP and greater PR and PTP at 6 months $(P<0.05)$ and 1 year $(P<0.01)$ respectively.

Conclusion: Surgical B and D are superior to surgical A and C respectively.

\section{Background}

Pulmonary atresia with intact ventricular septum (PA/IVS) is a kind of complex cyanotic congenital heart disease with a low incidence, accounting for only $1 \%$ to $3 \%$ of congenital heart disease (CHD)[1] ; however, it is a critically ill condition with high mortality.

Given a large anatomical variation of PA/IVS, and diverse surgical treatments in each center, there is still no unified standard[2,3]. Nevertheless, the reasonable choice of the initial surgery method is the key to improving the survival rate, which is also an important factor affecting the prognosis. PA/IVS is classified into type I, type II and type III according to Bull囚s right ventricular three-part classification[4]. Most heart centers choose different initial surgery schemes according to the degree of right ventricular development and presence or absence of right ventricular coronary artery dependence (RVDCC). However, due to the complexity and diversity of PA/IVS, the specific initial surgery methods are different. Since the initial surgery of type III PA/IVS is almost BT shunt, it is not included in this study.

This retrospective study aimed to investigate the effect of different initial surgical procedures on the prognosis of type I and type II PA/IVS, and to evaluate the therapeutic effect, thereby providing reliable clinical data for optimizing the selection of initial procedures for PA/IVS. 


\section{Methods}

\section{Clinical data and grouping}

A total of 100 children with type I and type II PA/IVS admitted to our hospital from January 2010 to December 2019 were selected, and 50 patients with type I and another 50 patients with type II were selected. Patients with type I were divided into group $A(n=25)$ and group $B(n=25)$, and those with type II were divided into group $C(n=25)$ and group $D(n=25)$. All patients had patent ductus arteriosus (PDA), atrial septal defect (ASD)/patent foramen ovale (PFO) and extensive tricuspid regurgitation. This study has been performed in accordance with the Declaration of Helsinki and was approved by the of Second Hospital of Hebei Medical University Ethics Committee and included Statement of Exemption of Informed Consent.

\section{Preoperative preparation}

Echocardiography was performed in all patients before surgery, and diagnostic cardiac catheterization and angiocardiography were performed in 36 patients. All patients were diagnosed as type I PA/IVS or type II PA/IVS without RVDCC. There were no significant differences in gender, age, weight, tricuspid vale $Z$ value (TZ), degree of tricuspid valve regurgitation (TR), McGoon ratio, oxygen saturation (SpO2), inner diameter of PDA, size of ASD or PFO between groups $A$ and $B$, and between groups $C$ and $D(P>0.05$ for all, Table 1).

\section{Surgical methods}

The initial surgery was performed in group A with surgical A and in group B with surgical B. Groups C and were treated with surgical C and D respectively. BT shunt is to set up a GORETEX artificial blood vessel between the brachiocephalic artery and the right pulmonary artery. According to the experience of our department, the type of the pipeline was selected based on the weight of the child. The $3 \mathrm{~mm}$ Gore-tex pipeline can be selected when the weight is less than $2.5 \mathrm{~kg}$. The Gore-tex pipeline with a diameter of 3.5 $\mathrm{mm}$ is selected for the newborn and within $3 \mathrm{~kg}$, and the $4 \mathrm{~mm}$ Gore-tex pipeline is selected for $3-7 \mathrm{~kg}$. In order to avoid hypoxia intolerance, PDA was ligated after BT shunt without extracorporeal circulation, which could stabilize blood oxygen saturation, and avoid blood flow conflicts from the right ventricle and PDA to the pulmonary artery. Mosaic therapy, which had achieved good clinical results[5], was as follows: Under direct vision, the pulmonary catheter was inserted into the pulmonary valve and the pulmonary artery anulus was expanded under the guidance of transesophageal echocardiography (TEE). According to our experience, the expansion is safer when the ratio of balloon diameter to pulmonary valve annulus diameter is $1: 1.3$. The changes of pulmonary artery pressure were observed immediately by TEE after balloon dilatation. If the pressure difference was still greater than $30 \mathrm{~mm} \mathrm{Hg}$, the balloon tube 1 2 times larger than the initial expansion balloon was selected for repeated expansion. Pulmonary valve incision was performed without extracorporeal circulation, that is, temporary occlusion of superior vena cava, inferior vena cava and distal main pulmonary artery, open atresia of pulmonary valve through main pulmonary artery incision under direct vision; transpulmonary annulus patch, that is, transvalvular patch 
from right ventricular outflow tract to pulmonary artery under cardiopulmonary bypass and removal of a small amount of right ventricular hypertrophy bundle. As for surgical $D$, which was on the basis of surgical C, artificial single pulmonary valve made of $0.1 \mathrm{~mm}$ thick GORETEX membrane was used according to the diameter of pulmonary valve anulus obtained by body surface area of children, so as to minimize pulmonary valve regurgitation.

Four groups of patients underwent the initial surgery according to the corresponding surgical methods. The MVT, length of ICU stay and perioperative mortality rate were recorded and statistically analyzed. The TZ, McGoon ratio, and tricuspid valve regurgitation in each group were recorded before surgery, at 1 month, 3 months, 6 months, and 1 year after surgery, and statistical analysis was performed. SpO2, PR and PTP were recorded and statistically analyzed at discharge, at 1 month, 3 months, 6 months and 1 year after surgery. During the follow-up period, the data of patients who had undergone surgical intervention or radical surgery were excluded th the corresponding time points. The survival rate and reoperation rate from 1 day to 1 year after surgery were recorded and statistically analyzed.

\section{Statistical analysis}

IBM SPSS Statistics22 statistical software was used for statistical analysis. One-sample KolmogorovSmirnov Test was used to determine normality of data. Measurement data are expressed as mean \pm standard deviation ( $x \pm s$ ). Differences in categorical variables between groups were tested using independent sample t test, Pearson chi-square test, and Continuity Correction. Kaplan-Meier method was used for calculating survival curve and reoperation curve, both of which were drawn by GraphPad Prism software, and a between-group comparison was performed using the log-rank test. $P<0.05$ indicated that the difference was statistically significant, and $\mathrm{P}<0.01$ indicated that the difference was highly significant.

\section{Results}

No statistically significant differences in the MVT, length of ICU stay, and perioperative mortality rate after surgery between group $A$ and group $B(P>0.05$ for all,Table 2$)$. The $P R$ was significantly greater in group $A$ than in group $B$ at 1 month after surgery $(P<0.05$,Table 7$)$. At 3 months after surgery, the TZ and McGoon ratio were lower in group $A$ than in group $B(P<0.05$, Table 3,Table 4), whereas the PR, PTP, and TR were significantly greater in group $A$ than in group $B(P<0.05$,Table 7,Table 8,Table 5). At 6 months after surgery, the SpO2 was lower in group $A$ than in group $B(P<0.05$,Table 6$)$, while the PTP was significantly greater in group $A$ than in group $B(P<0.01$,Table 8$)$. The PTP was greater in group $A$ than in group $B$ at 1 year after surgery $(P<0.05$,Table 8$)$. There was no statistically significant difference in the survival rate and re-intervention rate between group $A$ and group $B$ ( $P>0.05$,Fig.1,Fig.2,Fig.5). MVT and length of ICU stay were greater in group $C$ than in group $D(P<0.05$,Table 2$)$. At 1 month after surgery, the $P R$ was significantly higher in group $C$ than in group $D(P<0.01$,Table 7); at 3 months after surgery, the TR and the $P R$ in group $C$ were higher than those in group $D(P<0.05$,Table 5,Table 7). The McGoon ratio, Sp02, and PTP were lower in group $C$ than in group $D(P<0.05$,Table 4,Table 6,Table 8). At 6 months after surgery, 
the PR was higher in group $C$ than in group $D(P<0.05$,Table 7$)$, while the TZ and PTP were lower than those in group $D(P<0.05$,Table 3,Table 8). At 1 year after surgery, the PTP was significantly higher in group $D$ than in group $C(P<0.01$,Table 8$)$. There was no significant difference in survival rate between group $C$ and group $D(P>0.05$,Fig.4,Fig. 5 ), and the survival rate was $84 \%$ in group $C$ and $96 \%$ in group $D$. No significant difference was found in the re-intervention rate between group $C$ and group $D$ (P>0.05,Fig.3,Fig.5).

\section{Discussion}

PA/IVS has a low incidence but a high mortality rate, and great anatomical variation. Surgical programs are also diverse in each center, therefore, there is still no unified standard. Individualized surgical treatment is favourable, and the key step is to improve the early survival rate. According to our experience, few patients have one-stage radical operation; instead, most need staging surgery. For patients with mild and moderate right ventricular dysplasia and without RVDCC, right ventricular decompression should be performed as early as possible through initial surgery, thereby promoting the development of the right ventricle, improving the opportunity of biventricular repair in children[7,8], and eventually completing biventricular repair as early as possible[22].

Although much more surgical methods of right ventricular decompression are available for PA/IVS[914],surgical failure[15] and complications[16-19]are also universal. Hypoxemia is one of the most common complications for the following reasons: ( 1 ) Muscle edema of right ventricule, SpO2 in the early stage is not well maintained; ( 2 ) Hypoplastic right ventricle does not have sufficient pumping capacity to maintain the forward blood flow after the opening of pulmonary valve[20], and the pulmonary vascular bed pressure that has not been fully decreased in the neonatal period also limits the blood flow.

The measure to correct hypoxemia is to establish BT shunt. Nonetheless, it is still controversial whether decompression surgery is routinely combined with BT shunt.

Some studies have reported that BT shunt may lead to volume overload and left heart failure[6]. Our study believes that PDA is more likely to close when SpO2 becomes higher after right ventricular decompression, which has a great influence on the prognosis of PA/IVS. Therefore, PDA is routinely ligated and BT shunt surgery is added to ensure the stability and persistence of blood between systemic and pulmonary circulation.

In recent years, mosaic therapy has become a new approach for right ventricular decompression in the early stage of PA/IVS, which overcomes the shortcomings of interventional and surgical treatment, ${ }^{23}$ avoids the damage caused by cardiopulmonary bypass, with fast postoperative recovery, and could shorten the length of ICU stay.

Combined with mosaic therapy, surgical A has achieved good clinical results. Pulmonary valve incision without extracorporeal circulation is a simple procedure, which is free from extracorporeal circulation, with quick postoperative recovery. 
Compared with surgical A, surgical B also have achieved good surgical results, and there were no differences in MVT, length of ICU stay, and perioperative mortality rate between group A and group $B$ $(P>0.05$,Table 2). However, the mosaic therapy has several shortcomings, in which the puncture needle may be deviated from the center of the pulmonary valve, and the valve division is unevenly dilated, which finally reduces the function of the pulmonary valve. Due to tissue growth, adhesion, scar and other reasons after balloon dilatation, pulmonary valve will be reduced to varying degrees, hindering the growth of the right ventricle. The corresponding data showed that the PR was greater in group A than in group $B$ at 1 and 3 months after surgery $(P<0.05$,Table 7$)$.

The PTP was higher in group $A$ than in group $B$ at 3 months $(P<0.05$,Table 8$), 6$ months $(P<0.01$,Table 8$)$, and 1 year after surgery $(P<0.05$,Table 8$)$. Since pulmonary valve incision does not have the abovementioned disadvantages, surgical $B$ is better and more economical than surgical $A$, since it does not use intervention consumables.

Compared with surgical C, surgical D added an artificial pulmonary valve made of GORETEX membrane, which could reduce pulmonary valve regurgitation, protect right ventricular function, shorten MVT and length of ICU stay, promote right ventricular development, and improve the survival rate of initial surgery to a certain extent. The corresponding data showed that the PR was higher in group $C$ than in group $B$ at discharge $(P<0.05$,Table 7), and at 1 month $(P<0.01$,Table 7), 3 months $(P<0.05$,Table 7$)$, and 6 months after surgery $(P<0.05$,Table 7$)$. The MVT and length of ICU stay were longer in group $C$ than in group $D$, $(P<0.05$,Table 2). As for the causes of death in groups $C$ and $D$, right heart failure was the highest in group $\mathrm{C}$, which also indicated the advantages of artificial pulmonary valve in protecting the right ventricle.

Surgical $D$ also has shortcomings. Since the artificial pulmonary valve made by Goretex membrane will not grow with age, the incidence of PTP will increase as they get older.

This study has several limitations. First, we failed to detect the real differences between groups due to insufficient sample size. Second, the follow-up period afte initial surgery was insufficient to reflect the trend of the observation indexes, which may have introduced a certain bias on the statistical analysis results. In the future, studeis with larger samples and longer follow-up period should be conducted for further analysis. Some patients with radical conditions did not receive timely surgery, and some patients

who needed early reintervention fail to undergo timely surgery for various reasons, including large PTP or severe PR. It was related to the economic conditions and ideas of the guardians of the patients. Some parents could not afford medical treatment, and some parents believed that their children were in good living conditions and did not need reoperation. In addition, it is also associated with the conception of several surgeons that the older the child is, the higher the success rate of reoperation is.

\section{Conclusion}

Early active surgical treatment of PA/IVS is the key to improving the survival rate, and staged decompression is relatively stable, even if this may increase the rate of postoperative reintervention[21].The reasonable choice of the initial surgical strategy is an important factor affecting 
the prognosis. Taken together, surgical B is superior to surgical A for type I PA/IVS and surgical D is superior to surgical $C$ with respect to type II PA/IVS.

\section{Declarations}

\section{Ethics approval and consent to participate}

This study has been performed in accordance with the Declaration of Helsinki and was approved by the of Second Hospital of Hebei Medical University Ethics Committee and included Statement of Exemption of Informed Consent.

\section{Consent for publication}

Not applicable.

\section{Availability of data and materials}

The datasets used and analyzed during the current study are available from the corresponding author on reasonable request.

\section{Competing interests}

The authors declared that they have no conflict of interest or any other competing interest regarding results and/or discussion reported in the research.

\section{Funding}

None

\section{Authors $₫$ contributions}

All authors read and approved the manuscript for publication. HS, as the project administrator, contributed to data curation, investigation, methodology, resources, software, validation and literature review of the study. ZC contributed to conceptualization, formal analyses, resources, and literature review of the study and draft, reviewed, and edited the manuscript for intellectual content. All authors agree to be accountable for all aspects of work ensuring integrity and accuracy.

\section{Acknowledgements}

The authors are thankful to the surgical, medical, and non-medical staff of the Second Hospital of Hebei Medical University, China.

\section{References}


1. He X, Zhang $X$, Jing $H$, et al. Rare copy number variations might not be involved in the molecular pathogenesis of PA-IVS in an unselected Chinese cohort. Pediatr Cardiol, 2019, 40(4): 762-767.

2. Schneider AW, Blom NA, Bruggemans EF, et al. More than 25 years of experience in managing pulmonary atresia with intact ventricular septum. Ann Thorac Surg, 2014, 98(5): 1680-1686.

3. Wright LK, Knight JH, Thomas AS, et al. Long-term outcomes after intervention for pulmonary atresia with intact ventricular septum. Heart, 2019, 105(13): 1007-1013.

4. Bull C, de Leval MR, Mercanti C, et al. Pulmonary atresia and intact ventricular septum: a revised classification. Circulation, 1982, 66(2): 266-272.

5. Lin ZQ, Chen Q, Cao H, et al. Transthoracic balloon pulmonary valvuloplasty for treatment of congenial pulmonary atresia patients with intact ventricular septum. Med Sci Monit, 2017, 23: 4874.

6. Do N, Hill KD, Wallace AS, et al. Shunt failure-risk factors and outcomes: An analysis of The Society of Thoracic Surgeons Congenital Heart Surgery Database. Ann Thorac Surg, 2018, 105(3): 857-864.

7. Kotani Y, Kasahara S, Fujii Y, et al. A staged decompression of right ventricle allows growth of right ventricle and subsequent biventricular repair in patients with pulmonary atresia and intact ventricular septum. Eur J Cardiothorac Surg, 2016, 50(2): 298-303.

8. Cleuziou J, Schreiber C, Eicken A, et al. Predictors for biventricular repair in pulmonary atresia with intact ventricular septum. Thorac Cardiovasc Surg, 2010, 58(6): 339-344.

9. Lefort B, Saint-Etienne C, Soulé $\mathrm{N}$, et al. Perforation of the atretic pulmonary valve using chronic total occlusion (CTO) wire and coronary microcatheter. Congenit Heart Dis, 2019, 14(5): 814-818.

10. El Saiedi SA, Attia WA, Abd El-Aziz OM, et al. A perforation procedure for pulmonary atresia with intact ventricular septum: Egyptian experience and adaptations. Herz, 2018, 43(7): 633-641.

11. Rathgeber $S$, Auld B, Duncombe $S$, et al. Outcomes of radiofrequency perforation for pulmonary atresia and intact ventricular septum: a single-centre experience. Pediatr Cardiol, 2017, 38(1): 170175.

12. Lin ZQ, Chen Q, Cao H, et al. Transthoracic balloon pulmonary valvuloplasty for treatment of congenial pulmonary atresia patients with intact ventricular septum. Med Sci Monit, 2017, 23: 48744879.

13. Gupta SK, Juneja R, Saxena A. Central perforation of atretic pulmonary valve using coronary microcatheter. Ann Pediatr Cardiol, 2017, 10(3): 304-305.

14. Chen RHS, Chau AKT, Chow PC, et al. Achieving biventricular circulation in patients with moderate hypoplastic right ventricle in pulmonary atresia intact ventricular septum after transcatheter pulmonary valve perforation. Congenit Heart Dis, 2018, 13(6): 884-891.

15. El Shedoudy S, El-Doklah E. Transcatheter perforation of atretic pulmonary valve by the stiff end of a coronary wire in neonates with pulmonary atresia with intact ventricular septum: a solution in developing countries. J Saudi Heart Assoc, 2018, 30(3): 222-232.

16. Petit CJ, Qureshi AM, Glatz AC, et al. Technical factors are associated with complications and repeat intervention in neonates undergoing transcatheter right ventricular decompression for pulmonary 
atresia and intact ventricular septum: results from the congenital catheterisation research collaborative. Cardiol Young, 2018, 28(8): 1042-1049.

17. Bakhru S, Marathe S, Saxena M, et al. Transcatheter pulmonary valve perforation using chronic total occlusion wire in pulmonary atresia with intact ventricular septum. Ann Pediatr Cardiol, 2017, 10(1): 5-10.

18. Hasan BS, Bautista-Hernandez V, McElhinney DB, et al. Outcomes of transcatheter approach for initial treatment of pulmonary atresia with intact ventricular septum. Catheter Cardiovasc Interv, 2013, 81(1): 111-118.

19. Patil NC, Saxena A, Gupta SK, et al. Perforating the atretic pulmonary valve with CTO hardware: technical aspects. Catheter Cardiovasc Interv, 2016, 88(5): E145-E150.

20. Cho MJ, Ban KH, Kim MJ, et al. Catheter-based treatment in patients with critical pulmonary stenosis or pulmonary atresia with intact ventricular septum: a single institute experience with comparison between patients with and without additional procedure for pulmonary flow. Congenit Heart Dis, 2013, 8(5): 440-449.

21. Petit CJ, Glatz AC, Qureshi AM, et al. Outcomes after decompression of the right ventricle in infants with pulmonary atresia with intact ventricular septum are associated with degree of tricuspid regurgitation: results from the congenital catheterization research collaborative. Circ Cardiovasc Interv, 2017, 10(5): e004428.

22. Grant S, Faraoni D, DiNardo J, et al. Predictors of mortality in children with pulmonary atresia with intact ventricular septum. Pediatr Cardiol, 2017, 38(8): 1627-1632.

23. Lin ZQ, Chen Q, Cao H, et al. Transthoracic balloon pulmonary valvuloplasty for treatment of congenial pulmonary atresia patients with intact ventricular septum. Med Sci Monit, 2017, 23: 48744879.

\section{Tables}

Due to technical limitations, table 1 to 8 is only available as a download in the Supplemental Files section.

\section{Figures}




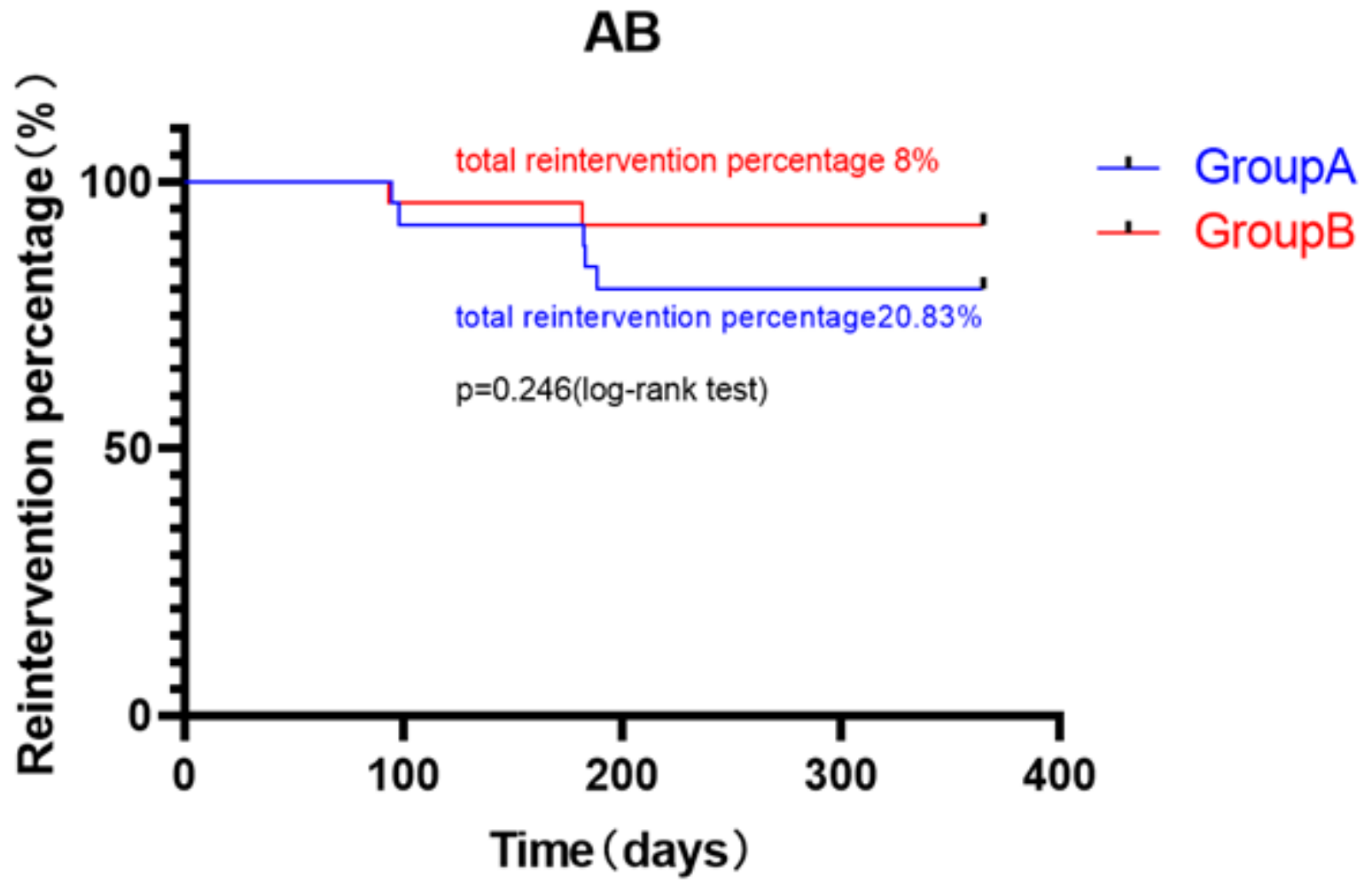

Numbers at risk

Group A 25

Group B 25

23

21

21

0

24

23

23

0

Figure 1

Kaplan-Meier curve analysis of reintervention percentage between group $A$ and $B$ 


\section{AB}

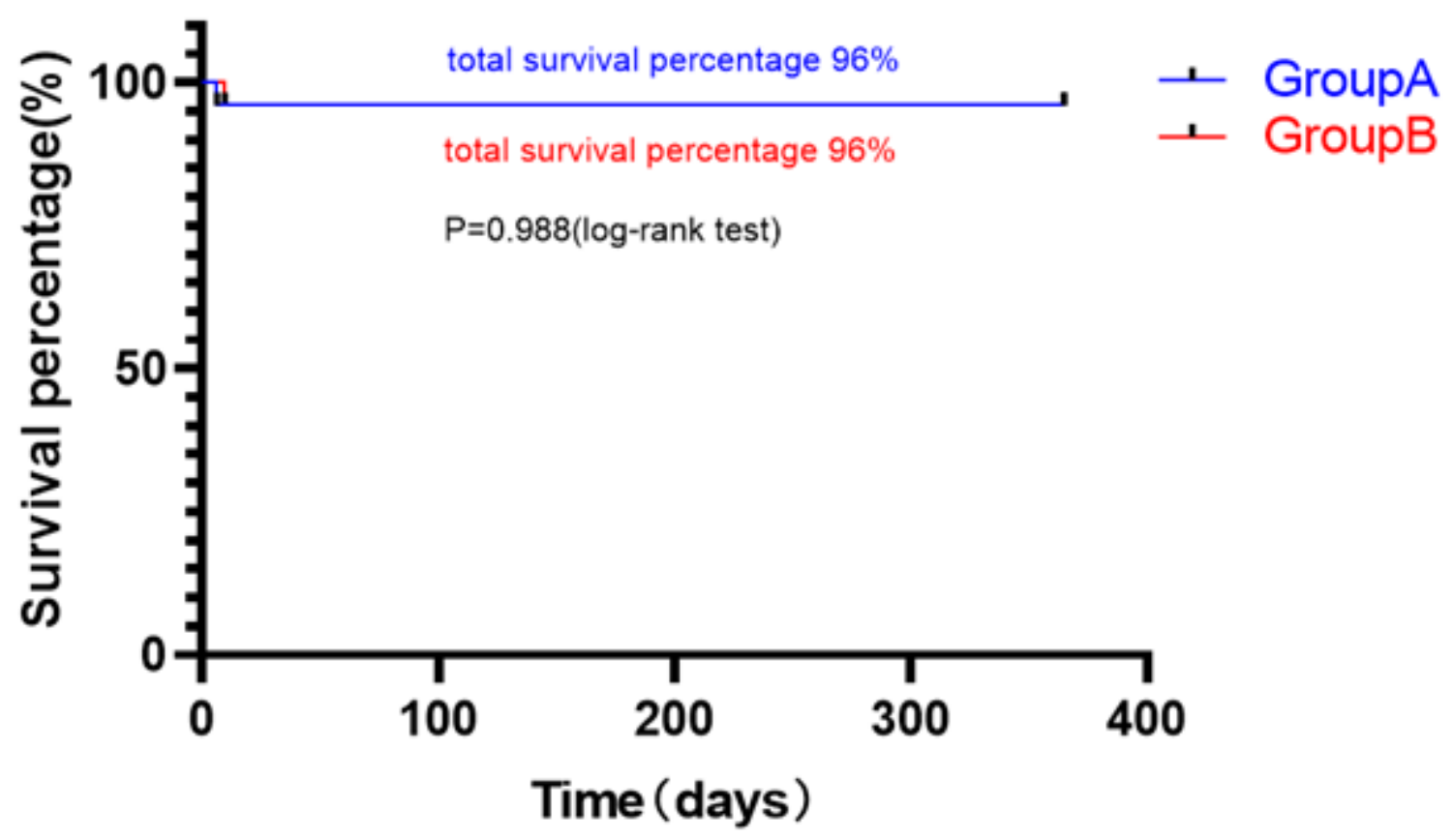

Numbers at risk

$\begin{array}{llllll}\text { Group A } & 25 & 24 & 24 & 24 & 0 \\ \text { Group B } & 25 & 24 & 24 & 24 & 0\end{array}$

Figure 2

Kaplan-Meier curve analysis of survival percentage between group A and B 
CD

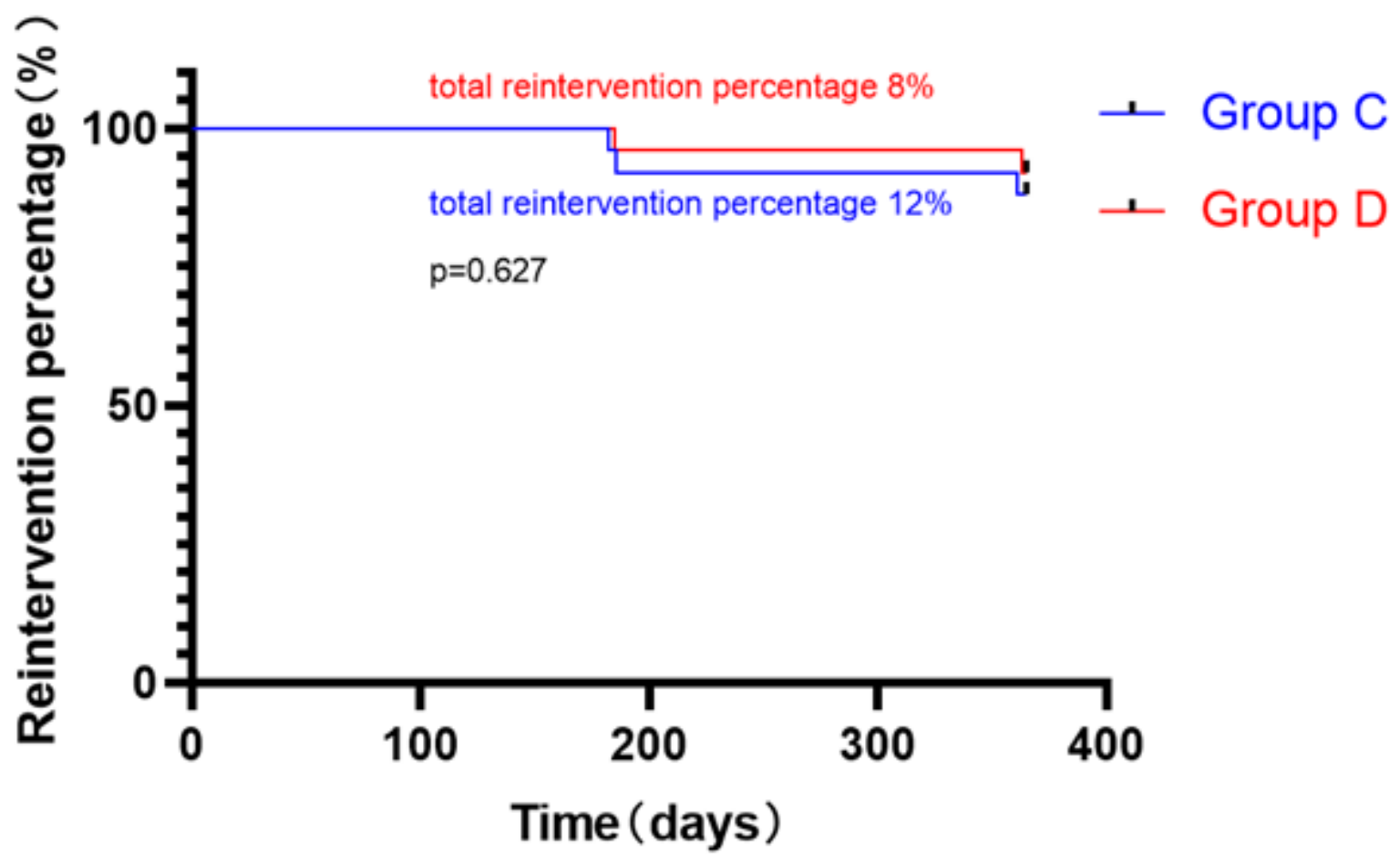

Numbers at risk

Group C 25

Group D 25

25

23

$23 \quad 0$

$25 \quad 24$

24

0

Figure 3

Kaplan-Meier curve analysis of reintervention percentage between group $C$ and $D$ 


\section{CD}

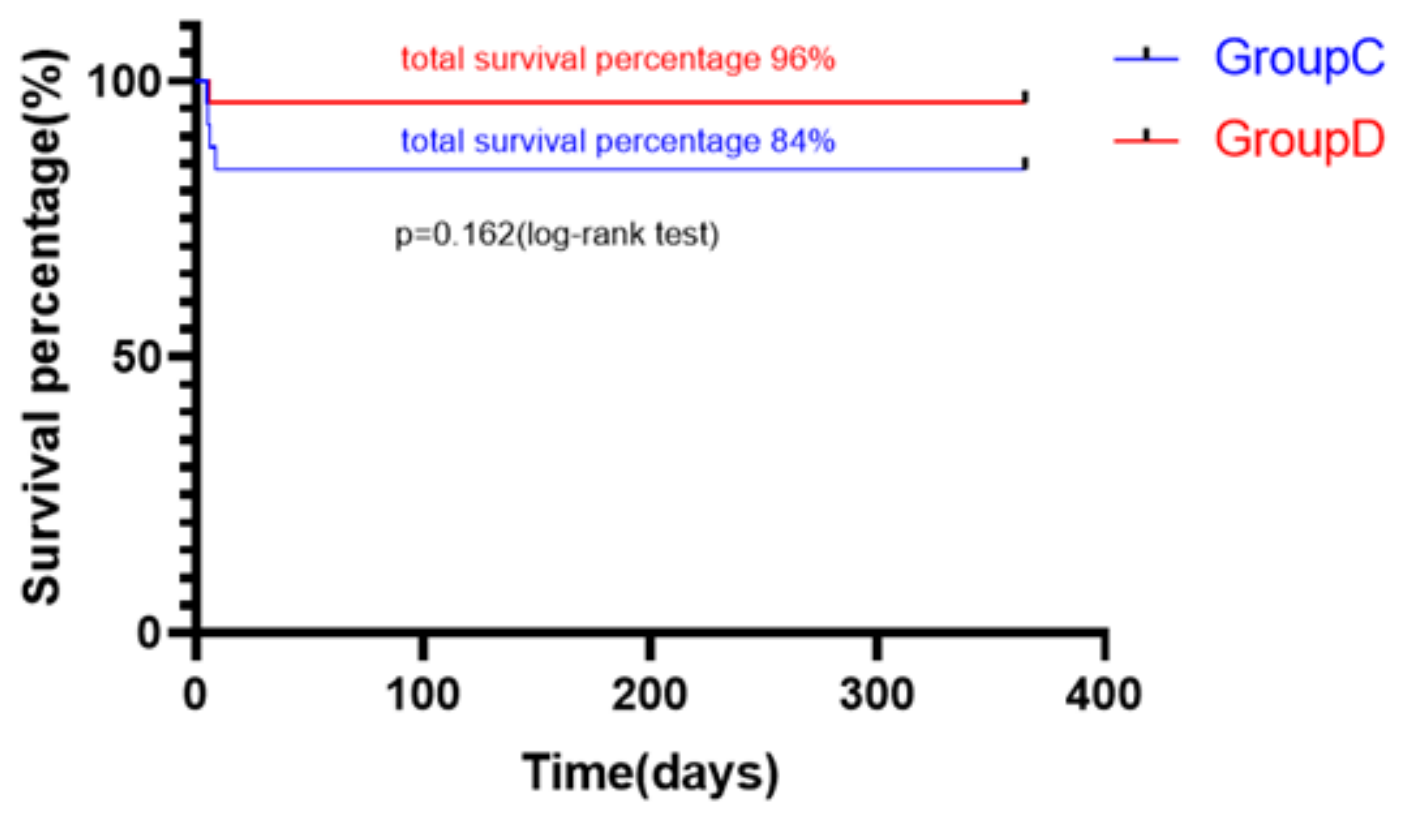

Numbers at risk:

$\begin{array}{llllll}\text { Group C } & 25 & 21 & 21 & 21 & 0 \\ \text { Group D } & 25 & 24 & 24 & 24 & 0\end{array}$

Figure 4

Kaplan-Meier curve analysis of survival percentage between group C and D 


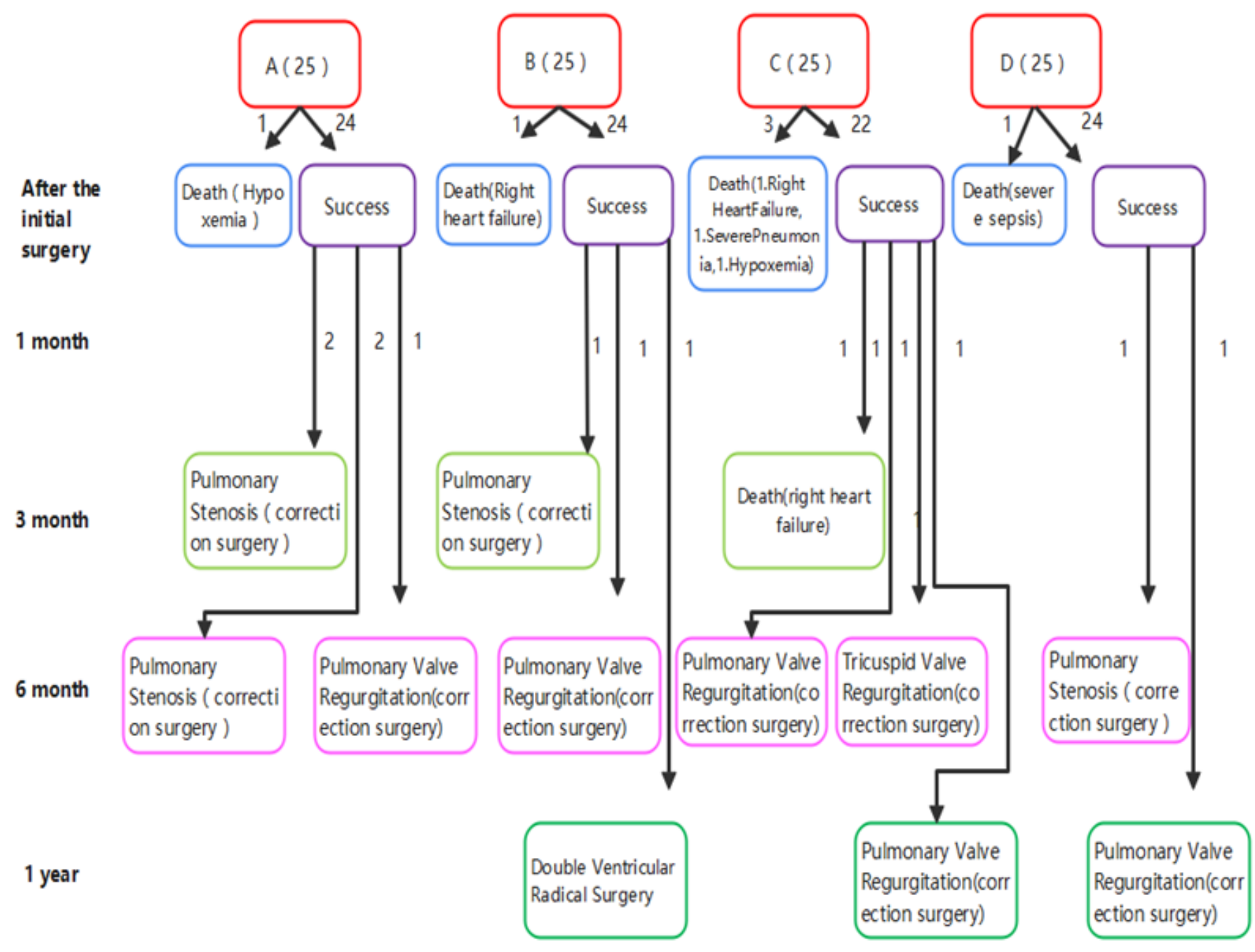

Figure 5

Flow chart displaying short-term outcomes of patients with different initial surgical procedures

\section{Supplementary Files}

This is a list of supplementary files associated with this preprint. Click to download.

- Tables.docx 